\title{
Efficacy of blood flow restriction exercise during dialysis for end stage kidney disease patients: protocol of a randomised controlled trial
}

\author{
Matthew J. Clarkson ${ }^{1}$, Steve F. Fraser ${ }^{1}$, Paul N. Bennett ${ }^{2,3}$, Lawrence P. McMahon ${ }^{4}$, Catherine Brumby ${ }^{4}$
} and Stuart A. Warmington ${ }^{1 *}$ (i)

\begin{abstract}
Background: Exercise during haemodialysis improves strength and physical function. However, both patients and clinicians are time poor, and current exercise recommendations add an excessive time burden making exercise a rare addition to standard care. Hypothetically, blood flow restriction exercise performed during haemodialysis can provide greater value for time spent exercising, reducing this time burden while producing similar or greater outcomes. This study will explore the efficacy of blood flow restriction exercise for enhancing strength and physical function among haemodialysis patients.

Methods: This is a randomised controlled trial design. A total of 75 participants will be recruited from haemodialysis clinics. Participants will be allocated to a blood flow restriction cycling group, traditional cycling group or usual care control group. Both exercising groups will complete 3 months of cycling exercise, performed intradialytically, three times per week. The blood flow restriction cycling group will complete two 10-min cycling bouts separated by a 20-min rest at a subjective effort of 15 on a 6 to 20 rating scale. This will be done with pressurised cuffs fitted proximally on the active limbs during exercise at 50\% of a pre-determined limb occlusion pressure. The traditional cycling group will perform a continuous 20-min bout of exercise at a subjective effort of 12 on the same subjective effort scale. These workloads and volumes are equivalent and allow for comparison of a common blood flow restriction aerobic exercise prescription and a traditional aerobic exercise prescription. The primary outcome measures are lower limb strength, assessed by a three repetition maximum leg extension test, as well as objective measures of physical function: six-minute walk test, 30-s sit to stand, and timed up and go. Secondary outcome measures include thigh muscle cross sectional area, body composition, routine pathology, quality of life, and physical activity engagement.
\end{abstract}

Discussion: This study will determine the efficacy of blood flow restriction exercise among dialysis patients for improving key physiological outcomes that impact independence and quality of life, with reduced burden on patients. This may have broader implications for other clinical populations with similarly declining muscle health and physical function, and those contraindicated to higher intensities of exercise.

Trial registration: Australian and New Zealand Clinical Trial Register: ACTRN12616000121460.

Keywords: End-stage kidney disease, Dialysis, Exercise, Strength, Physical function, Blood flow restriction exercise

\footnotetext{
* Correspondence: stuart.warmington@deakin.edu.au

'Institute for Physical Activity and Nutrition, School of Exercise and Nutrition

Sciences, Deakin University, 221 Burwood Highway, Burwood 3125, Australia

Full list of author information is available at the end of the article
} 


\section{Background}

End-stage kidney disease (ESKD) is the 5th stage of chronic kidney disease, characterised by a failure of the kidneys to adequately filter blood [1]. ESKD is estimated to affect as many as 2 million people worldwide, approximately half of whom do not receive adequate treatment $[2,3]$. Treatment requires either transplantation, or if transplantation is unavailable patients are solely reliant on regular dialysis [4]. Largely due to the regularity of dialysis as a treatment, ESKD is among the most common reasons for hospitalisation [2]. However, despite dialysis being considered one of the most efficacious means of treating ESKD, it does not guarantee that life can be sustained, with 12 month mortality rate following initiation of dialysis estimated to be $10-12 \%$ [5].

ESKD patients suffer from anaemia, fatigue, muscle atrophy, decreased aerobic capacity, reduced participation in physical activity, increased risk of falls, and decreased quality of life [4, 6-8]. Patients with ESKD also demonstrate significant decline in physical function and tasks reflective of activities of daily living [9-13]. This diminished physical function leads to a significant loss of independence with $95 \%$ of people with ESKD not fully independent and in need of assistance with at least one activity of daily living (ADL) [14]. This is more significant considering that an inability to independently complete ADLs is a major predictor of mortality for ESKD [14]. Exercise is well tolerated among patients with ESKD and is an efficacious method for ameliorating these adverse symptoms characteristic of ESKD [15-18].

Heterogenous methodology, prescription and reporting among ESKD exercise studies make it difficult to draft guidelines on the dosage and mode of exercise training to derive the best outcomes for ESKD patients $[8,19]$. The current guidelines for exercise training with ESKD recommend it encompasses aerobic (4 sessions; 45 mins), resistance (2 sessions; 20 mins), and flexibility (7 sessions; 10 mins) exercise [20]. The cumulative time commitment for these recommendations is substantial, and would require training on both dialysis (during first $2 \mathrm{~h}$ of dialysis) and non-dialysis days [20]. However, most randomised controlled trials examining exercise regimens in ESKD have only utilised aerobic training, most commonly stationary cycling [20]. Aerobic exercise training alone does not traditionally increase strength and hypertrophy, nor produce significant improvements in physical function, all of which are needed among this population [21].

Adherence to exercise training programs, regardless of aerobic, resistance or combined modalities, is also poor among ESKD patients, with more than $20 \%$ choosing not to participate, citing issues such as lack of time, lack of energy, too much trouble, and resistance training being too difficult [22]. Of those that chose to participate in exercise training, the dropout rate was more than $25 \%$ for programs utilising exercise training on non-dialysis days [22]. While there is still an increased dropout rate among patients with ESKD when exercise is completed intradialytically (approximately 17\%) [22], it is lower than that seen on non-dialysis days. This suggests that intradialytic exercise is still preferable with regards to participant retention than exercise on non-dialysis days.

Resistance training poses further complications in the setting of a dialysis unit as the required equipment is cumbersome, and poses a high risk for cross-infection. Less cumbersome washable elastic resistance bands may be one option [23]. Exercise training during dialysis also needs to be completed in the first $2 \mathrm{~h}$ of dialysis sessions [24]. Therefore, finding a solution that may provide improvements in all key outcomes as efficiently as possible, allowing for completion during dialysis would be preferable.

Blood flow restriction exercise training uses pressurised cuffs fitted proximally on the active limbs during exercise training, and can elicit the above benefits at lower exercise intensities than non- blood flow restriction training $[25,26]$. Blood flow restriction may be one method for achieving outcomes reflective of both resistance, and aerobic exercise training while providing an efficient, simple exercise prescription model in the dialysis setting. Blood flow restriction aerobic exercise training has been shown to increase muscle size and strength more than equivalent-intensity non-blood flow restriction aerobic exercise training, while still providing comparable or greater improvements in cardiovascular fitness $[25,27,28]$.

While blood flow restriction exercise training is used predominately in single joint isolation resistance training exercises such as knee extension, it has also been applied during aerobic exercise training such as walking or cycling [25, 29-32]. Common blood flow restriction aerobic exercise training protocols utilise short exercise durations of 10 to $20 \mathrm{~min}$ [25, 32-34]. Such studies utilising blood flow restriction with aerobic exercise training have demonstrated increases in lower limb muscle volume and strength of up to $8 \%$ and $16 \%$, respectively $[25,32,33]$. However, it should be noted that without blood flow restriction, low-intensity aerobic exercise training is generally insufficient to increase muscle volume or strength, unless there is previous muscle atrophy or the initial level of participant physical activity is extremely low [35]. Even in such instances, improvements in muscle volume and strength are unlikely to be of similar magnitude as seen with blood flow restriction [35].

Regardless of the presence of blood flow restriction, aerobic exercise training improves aerobic fitness provided that the exercise is of sufficient intensity [36]. Indeed, studies have demonstrated that low-to-moderate intensity blood flow restriction aerobic exercise training 
is able to increase aerobic capacity when exercising at intensities equivalent to $40 \%$ of peak exercise oxygen consumption ( $\mathrm{V}_{2}$ peak) [32, 33, 37].

Studies examining the haemodynamic responses to blood flow restriction identify blood flow restriction aerobic exercise training as having lower haemodynamic stress than high intensity resistance training, while providing similar outcomes [38-40]. As such, blood flow restriction is purported to be a potentially beneficial addition to exercise for clinical populations who may be contraindicated to high-intensity resistance training. While blood flow restriction exercise is a novel addition to exercise among ESKD patients, pilot data from this laboratory (unpublished) indicates that haemodynamic responses (systolic blood pressure, diastolic blood pressure, mean arterial pressure, heart rate) do not differ markedly between low-intensity blood flow restriction and non-blood flow restriction aerobic exercise both performed during haemodialysis in this population.

Aerobic capacity and muscle strength are both key physiological factors underpinning physical function [41] and both can improve with blood flow restriction aerobic exercise training. As declines in physical function, muscle strength, and aerobic capacity, as well as accelerated muscle atrophy are common among patients with ESKD, blood flow restriction aerobic exercise training may present an opportunity to increase the efficiency of time spent exercising for this population.

This study will examine the efficacy of blood flow restriction aerobic training among patients with ESKD on haemodialysis (HD). We hypothesise that blood flow restriction aerobic exercise training will improve physical function, muscle strength, and muscle size among patients with ESKD beyond that of traditional aerobic exercise training.

\section{Methods \\ Design}

This is a 12-week randomised controlled trial consisting of three groups randomised to exercise or usual care (Fig. 1). Participants will be randomised to one of three groups: a blood flow restriction cycling exercise training group (BFR-C), a non-blood flow restriction cycling exercise training group (CYC), or a non-exercising, usual care control group $(\mathrm{CON})$.

\section{Sample size calculation}

The most relevant outcome for blood flow restriction training studies is knee extensor muscle strength, and this was used as the main primary outcome measure for calculating required sample size. Based on data from previous blood flow restriction research [25, 29, 32, 33], and previous exercise for ESKD research [18, 42, 43], the projected percentage changes in knee extensor muscle strength for each of the three groups are $\mathrm{CON}=-3 \%$, CYC $=2 \%$, BFR-C $=12 \%$. The common standard deviation within group, based on the prior research was determined to be 15 . Thus, to achieve a power of $80 \%$ at an $\alpha$ level of $0.05,20$ participants per group will be required to detect a difference between the expected means for percentage strength improvement. However, based on previous work conducted by this research group a conservative attrition rate of $20 \%$ is expected over 3 months due to voluntary patient withdrawal, death, additional hospitalisation, transfer to another facility, or patients receiving transplants [23]. Thus, a total of 75 participants will be recruited and randomly allocated to one of the three groups.

\section{Recruitment and screening of participants}

Participants will be recruited through recommendation and referral from treating physicians at a number of dialysis clinics linked to a major public hospital service in a large metropolitan Australian city. Additionally, nurse unit manager recommendations and fliers briefly outlining the study posted within the dialysis clinics will be used to help identify prospective participants. Prospective participants will be screened initially face-to-face or via telephone by a member of the research team. Following this, confirmation from the treating physician will be obtained if not implied by referral. Participants will be required to provide written, informed consent prior to participation in the study.

\section{Inclusion and exclusion criteria}

Eligible participants will be male and female stable chronic (>3 months) haemodialysis patients aged between 18 and 80 years. Participants will be deemed medically eligible by their treating physician before participation in the present study. Participants will be excluded if they do not understand English and are unable to complete or comprehend the surveys or study documents; if within the previous 12 weeks they have participated in regular physical activity or sport (>150 min.wk. ${ }^{-1}$ ) of moderate or greater intensity [44], or structured resistance training (> 1 session.wk. ${ }^{-1}$ ); if they have symptomatic peripheral vascular disease, limb ischemia, untreated symptomatic cardiovascular disease, or any other absolute contraindications to exercise training (such as musculoskeletal factors or neurological conditions) that may affect their ability to perform physical assessments or exercise training protocols in the present study; if they are currently smokers; or if they are pregnant or have required hospitalisation for non-dialysis reasons in the 4 weeks prior to the study's commencement. Patients will also be deemed unable to exercise during individual dialysis sessions if they present with fluid overload (> 5\% above dialysis base weight) as this can cause reduced cardiovascular reserve [45]. Similarly, if patients have systolic 


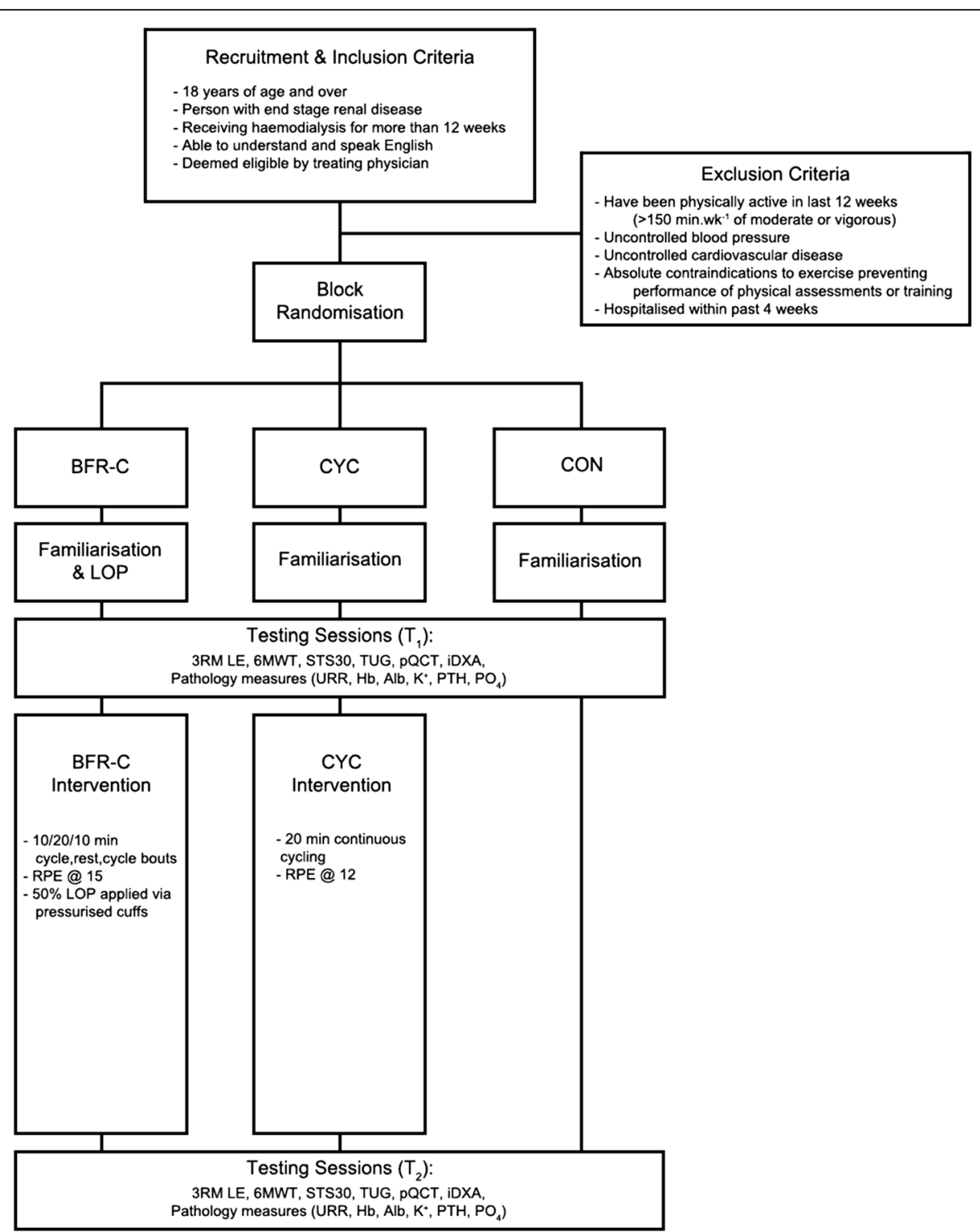

Fig. 1 Study Flow Chart. BFR-C - Blood flow restriciton cycling group; CYC - Traditional cycling group; CON - Usual care control group; LOP - Limb occlusion pressure; 3RM LE - Three-repetition Maximum Leg Extension; 6MWT - 6 min walk test; STS30-30-s sit-to-stand test; TUG - Timed up and go; pQCT - Peripheral Quantitative Computed Tomography; iDXA - Dual X-ray Absorptiometry; URR - Urea Reduction Ratio; Hb - Haemoglobin; Alb - Albumin; $\mathrm{K}^{+}$- Potassium; PTH - Parathyroid Hormone; PO4 - Phosphate; RPE - Rating of perceived exertion

blood pressure > $180 \mathrm{mmHg}$, or diastolic blood pressure $<90 \mathrm{mmHg}$ prior to exercise they will be deemed unsuitable for exercise on that day (excluding the first blood pressure reading before dialysis, which is known to be unreliable) [46]. Of clinical concern is the known propensity for HD patients to become hypotensive (potentially more so post-exercise) during dialysis [47]. Although this is uncommon and our pilot data indicates this is not exacerbated by blood flow restriction, to ensure the safety of all patients they will be monitored for chest pain/discomfort, dyspnoea, lower limb pain, symptoms of severe hyper- or hypotension, and other signs of adverse events, which will be reported to the ethics committee of both the treating hospital and Deakin University.

\section{Randomisation}

Randomisation will be conducted at a participant level prior to familiarisation and baseline testing, and will randomise participants by blocks of 4 via a computergenerated random number sequence by an independent researcher. Blinding of intervention group to clinical staff and participants will not be possible once the intervention commences. 


\section{Exercise intervention}

Exercise training sessions will occur intradialytically for all exercising participants and will be completed within the first $2 \mathrm{~h}$ of the session to avoid loss of exercise training quality $[24,48]$. All sessions will be supervised by a member of the research team. Exercise training sessions will consist of cycle exercise for both BFR-C and CYC groups. Both BFR-C and CYC groups will complete a total volume of 20 min cycling at a relative intensity for each participant to be dictated by rating of perceived exertion (RPE), similar to previous exercise and ESKD studies $[17,49,50]$. This will also be compared to measurements of percentage age-predicted maximum heart rate $\left(A P H R_{\max }\right)$ in order to determine if targeted RPE needs to be adjusted to account for potential discrepancies between patient subjective intensity and objectively observed intensity. If participants are unable to maintain the prescribed cadence at this workload for the full duration, they will be instructed to complete as much of each cycling bout as possible.

\section{Age-predicted maximum heart rate}

$\mathrm{APHR}_{\text {max }}$ will be used as a relative indicator of intensity during exercise training sessions. Approximately 50-70\% of participant's age-predicted $\mathrm{APHR}_{\max }$ will be used as an objective target to indicate whether participants in BFR-C or CYC are exercising at the prescribed intensity for this study. This will be achieved using a fingertip heart rate monitor and pulse oximeter (CMS50, Contec Medical Systems Co., Ltd., Qinhuangdao, Hebei Province, China) placed on the second or third finger of the participant's hand on the contralateral limb to their arteriovenous fistula. This will be measured and recorded during the final minute of each period of active cycling within a session to correspond with measures of RPE.

\section{Blood flow restriction cycling group}

The BFR-C group will complete an intermittent protocol of 10 min cycling, 20 min rest, 10 min cycling. During these sessions, participants in the BFR-C group will have a blood flow restriction cuff fitted to the proximal end of each thigh, which will be inflated continuously at $50 \%$ of a pre-determined limb occlusion pressure (LOP) throughout the full duration of cycling. Participants will be required to cycle at an RPE of 15 ('Hard' on the Borg 6 to 20 scale [51]), expected to be equivalent to at least $60 \%$ of $\mathrm{APHR}_{\max }$ for each $10 \mathrm{~min}$ cycling period. The prescribed volume and intensity represents a balance between prior blood flow restriction protocols and those utilised in the aerobic training components of HD studies, while accounting for potentially very low levels of physical function among the participants $[6,8,52]$.

\section{Blood flow restriction procedure}

Blood flow restriction will be applied using an automatic tourniquet system (A.T.S 3000, Zimmer Inc., OH, USA). The ATS produces a defined, controlled pressure via adjustable pneumatic cuffs $(52 \mathrm{~cm}$ long, $10.5 \mathrm{~cm}$ wide; bladder length $45 \mathrm{~cm}$, bladder width $8 \mathrm{~cm}$ ). The pressure is regulated to control for changes in muscle contractile pressures under the cuff in such a way that the occlusive pressure applied to the limb is constant.

The cuffs will be applied to the proximal aspect of the thigh prior to commencing exercise, and gradually inflated, as detailed by Abe et al. (2006) [25]. Blood flow restriction pressure will be set at $50 \%$ of pre-exercise resting LOP. LOP will be calculated during a familiarisation session for participants allocated to the BFR-C group using a digital plethysmograph (Pulse Sensor, Zimmer ATS 3000) applied to the second toe [40, 53]. A seated or recumbent posture will be used for LOP as it most closely matches the posture that will be used during the exercise training sessions. To determine LOP, the pneumatic cuffs are inflated to the point at which the plethysmograph can no longer detect blood flow to the toe (blood flow completely occluded). This will be done for each leg and repeated to ensure accuracy $(<20 \mathrm{mmHg})$, and an average of these measures will be taken. The 50\% LOP that is to be applied during exercise training sessions does not fully occlude the limb in this manner, and allows for continuous blood flow during exercise.

\section{Cycling group}

The CYC group exercise training sessions will require 20 min continuous cycling at an equivalent intensity to the BFR-C group. However due to the absence of blood flow restriction, the equivalent intensity is often reported to have a lower RPE and mildly reduced heart rate response, regardless of exercise modality [38, 54, 55]. As such, the target RPE will be 12 ('light to somewhat hard' on the Borg 6 to 20 scale [51]) for the CYC group, which is expected to equate to at least $50 \% \mathrm{APHR}_{\max }$. This intensity is recommended for intradialytic aerobic exercise in the Exercise and Sport Science Australia (ESSA) position statement on exercise for chronic kidney disease [20]. This volume is reflective of protocols used by other studies examining exercise training among patients with ESKD, and represents a "normal" exercise training prescription for patients with ESKD $[22,56]$.

\section{Progression}

In order to ensure that training sessions maintain a sufficient intensity for all exercising participants, resistance applied to the pedals, cadence, or a combination of both variables will be adjusted on a session-by-session basis by a trained exercise physiologist such that the required RPE target (indicative of sufficient subjective intensity) is 
achieved. This accounts for any illness or excessive fatigue that may affect exertion during any given session. A rolling two-week average of RPE will be monitored (on the 6-20 Borg's RPE scale [51]) to ensure progressive overload is being achieved over the course of the 3month intervention.

\section{Usual care control group}

Participants allocated to the usual care control group will receive no additional access to exercise training and will receive minimal advice regarding exercise training throughout the study. Participants randomised to the usual care control group will be given access to an accredited exercise physiologist member of the research team, free of charge if they would like to discuss the benefits of commencing exercise training upon completion of the study.

\section{Data collection}

Data collection will occur at baseline $\left(\mathrm{T}_{1}\right)$ and 12 weeks $\left(\mathrm{T}_{2}\right)$, with testing to be completed at the treating HD unit prior to the commencement of the participants' HD session. Personal information, including relevant medical history will be collected prior to the initiation of testing or intervention upon receiving participant consent. This will include patient characteristics of age, gender, time in months that the participant has been receiving HD (dialysis vintage), basic anthropometric measures (height, weight, and BMI), relevant comorbidities and medications, transplant status, and type of dialysis access. Some or all of which may be taken, with approval, from the case file at the HD unit. The remaining data collected will include all measures of muscle function and physical function. Body composition scans will be performed on a non-dialysis day, separate from other testing procedures for participants who opt-in to this aspect of testing. Additional pathology measurements will include haemoglobin, albumin, potassium, parathyroid hormone, phosphate, urea reduction ratio (URR), creatine kinase and lactate. These measures will occur as part of routine pathology tests where possible, or will be requested out-of-cycle to coincide with the necessary period of the training intervention. Data will be coded and stored securely at Deakin University. Data collected from those who withdraw from the study will not be used for statistical analyses. This has been accounted for when completing the power analysis, with an additional $20 \%$ added to the target sample size. Attrition may be for a number of reasons, which will be ascertained upon withdrawal where possible.

\section{Primary outcome measures} Lower-limb muscle strength

Maximal lower limb muscle strength will be determined using three-repetition maximum (3RM) knee extension
(HS-LE, Life Fitness, Victoria, Australia) [57]. A 3RM knee extension test measures the maximum weight that can be lifted for three consecutive repetitions of a knee extension exercise with good technique and full range of motion. The 3RM result will be input into a formula to derive equivalent one-repetition maximum for each participant [58].

\section{Physical function}

Objective physical function will be measured using the 30-s sit to stand test (STS30), the timed up and go test (TUG), and the six-minute walk test (6MWT).

The STS30 measures lower limb muscle strength and function and requires participants to stand from a chair and then return to the seated position as many times as possible in $30 \mathrm{~s}$ [59]. The test has also displayed strong comparative validity to lower extremity $1 \mathrm{RM}$ strength testing, particularly among community-dwelling older adults [59]. It has also demonstrated a high level of reliability among ESKD patients undergoing HD $(\mathrm{ICC}=0.93)$ [60].

The TUG is a measure of dynamic balance and mobility requiring participants to stand from a chair, walk to and then around a cone placed $3 \mathrm{~m}$ away, walk back to the chair, and sit back down as quickly as possible. This test will be repeated three times, and an average time taken for accuracy purposes [61]. The TUG strongly reflects gait speed among older adults, and has shown strong test retest reliability not only among older adults, but in ESKD patients undergoing HD (ICC $=0.91-0.96)$ [62-64].

The 6MWT is a self-paced assessment of aerobic capacity, and a strong index of all cause morbidity in older adults [65]. It requires participants to walk laps of a straight $30 \mathrm{~m}$ course with the objective to cover as much distance as possible in the six-minute duration [65]. The 6MWT is a reliable measure for patients with ESKD (ICC $=0.93-0.96)[60,66]$. It has also been validated against $\dot{\mathrm{V}}_{2}$ max for patients with ESKD, with moderate strength coefficients of correlation $(r=0.56-0.73)[11,67]$.

\section{Secondary outcome measures \\ Muscle cross-sectional area and body composition}

Total and regional body composition (lean mass, fat mass and percentage fat mass) will be assessed using iDXA (Lunar iDXA, GE Healthcare, Madison, WI). Muscle cross-sectional area around the femur diaphysis at $25 \%$ and $50 \%$ of femur length will be assessed using pQCT (XCT 3000, Stratec Medizintechnik, BadenWürttemberg, Germany), allowing for knee flexor and extensor musculature to be measured. Height and body mass will be assessed using a portable stadiometer and scales respectively.

\section{Pathology measures}

Pathology measurements will be taken during routine collections completed by the treating hospital. The 
exercise intervention will be timed such that these routine pathology measures occur within the 4 weeks prior to the commencement of the first training session (or prior to the 3 month usual care monitoring period for the $\mathrm{CON}$ ). These additional measures will include haemoglobin, albumin, potassium, parathyroid hormone, and phosphate. URR will also be measured by the treating hospital as a part of patients' quarterly pathology tests, providing it is within the 4 weeks prior to patients commencing their exercise intervention. If this cannot be achieved in a timely manner, an additional measure of URR will be requested for a single dialysis session prior to the commencement of the exercise intervention.

Two additional measures of URR, creatine kinase and lactate will also be required outside of the normal regimen for patients in either of the exercise training intervention groups, once during the first week of the exercise training intervention, and again during the final week of the exercise training intervention. An additional out-of-cycle measure of URR will also be required by participants in the usual care control group, unless this is scheduled to be completed within 2 weeks following their 3-month monitoring period for this study as a part of their quarterly routine pathology test. All measures are conducted at the beginning and upon completion of a single dialysis session to establish the difference in each marker over the course of that session. This will allow analysis of the impact of exercise and specific exercise intervention types (BFR-C and CYC) on dialysis adequacy, as well as providing objective proof that there is no major tissue damage as a result of the intervention.

\section{Physical activity levels}

The Community Healthy Activities Model Program for Seniors (CHAMPS) physical activity survey will be used to assess participation in a comprehensive list of low, moderate and vigorous physical activities [68]. This questionnaire will provide valuable information about changes in physical function and physical activity behaviour over the course of the study.

\section{Symptom related quality of life}

The POS-S Renal questionnaire will be given to patients during the familiarisation session to assess their quality of life in direct relation to the symptoms experienced as a part of their condition [69]. This questionnaire provides valuable feedback about any changes experienced by patients regarding quality of life and disease-specific symptoms as a result of the intervention.

\section{Statistical analysis}

All statistical analyses will be performed using SPSS 22.0 (IBM Corp, Chicago IL, United States of America). Participant characteristic and demographic data will be presented using descriptive statistics, and compared using independent t-tests for continuous variables and chisquare tests for categorical variables. The distribution of data will be assessed for normality with a Shapiro-Wilks test $(P>0.05)$. Non-normally distributed data will be compared using the Friedman non-parametric test. Otherwise, comparisons between groups for all continuous primary and secondary variables measured will be made using one-way analysis of variance (ANOVA). Main effects will be analysed using a Tukey post hoc test. A significance level of $P<0.05$ will be adopted for all statistical tests. All data will be presented as means \pm SEM unless stated.

\section{Discussion and conclusion}

This study is the first to explore the efficacy of blood flow restriction aerobic exercise training among patients with ESKD. Additionally, it is one of the first to explore a battery of common clinical measures or physical function following a blood flow restriction aerobic exercise training program. The main objective of the study is to determine the effectiveness of blood flow restriction aerobic exercise training for improving the strength and physical function among patients with ESKD.

While exercise during dialysis is not a novel concept in the broader literature, the current exercise recommendations present a significant time and physical burden for patients with ESKD and does not have an established role in clinical practice. Therefore, this study is important given the well documented deficits in strength and physical function for patients with ESKD in conjunction with the rarity with which any exercise is adopted not only intradialytically, but in the broader ESKD population. It may also be valuable to include measures related to vascular stiffness (e.g. pulse wave velocity and vascular-related microRNAs), as this has also been shown to improve with exercise training, and may be enhanced with the addition of blood flow restriction [34, 70, 71].

It is expected that this study will provide an improved understanding of the role that blood flow restriction aerobic exercise training can play in providing an efficacious, time-efficient modality of intradialytic exercise for patients with ESKD, which caters to the population's lower initial level of physical function and overall conditioning.

\footnotetext{
Abbreviations

6MWT: 6-min walk test; ADL: Activity of daily living; APHR max: Age-predicted maximum heart rate; BFR-C: Blood flow restriction cycling group; CHAMPS: Community healthy activities model program for seniors questionnaire; CON: Usual care control group; CYC: Traditional cycling group; ESKD: End-stage kidney disease; HD: Haemodialysis; iDXA: Dual X-ray absorptiometry; LOP: Limb occlusion pressure; PQCT: Peripheral quantitative computed tomography; RPE: Rating of perceived exertion; STS30: 30-s sit to stand test; TUG: Timed up and go test; URR: Urea reduction ratio; $\dot{\mathrm{V}} \mathrm{O}_{2}$ peak: Peak oxygen uptake
} 


\section{Acknowledgements}

Not applicable.

\section{Funding}

Facilities provided jointly by Eastern Health and Deakin University. This study was supported by funds made available from the School of Exercise and Nutrition Sciences, Deakin University.

\section{Availability of data and materials}

Not applicable.

\section{Authors' contributions}

MJC, SAW, SFF, and PNB developed the study concept and initiated the project. MJC, SAW, SFF, PNB, LPM, and CB provided significant input into the development of the protocol. MJC drafted the manuscript. MJC will implement the protocol and oversee collection of the data. MJC, SAW, SFF, PNB, LPM, and CB read, edited and approved the final manuscript.

\section{Ethics approval and consent to participate}

This study was performed in accordance with the Declaration of Helsinki (1975). The design and conduct of this study was approved by the human research ethics committees of both Deakin University (DUHREC 2016-223) and Eastern Health (E05-2016). Participants will be provided with plain language information packages that detail all aspects of their potential involvement in the study, as well as consent forms they will be required to read, complete and return to a member of the research team. This study has been registered with the Australian and New Zealand Clinical Trials Register (ANZCTR): ACTRN12616000121460

\section{Consent for publication}

Not applicable.

\section{Competing interests}

The authors declare that they have no competing interests.

\section{Publisher's Note}

Springer Nature remains neutral with regard to jurisdictional claims in published maps and institutional affiliations.

\section{Author details \\ ${ }^{1}$ Institute for Physical Activity and Nutrition, School of Exercise and Nutrition Sciences, Deakin University, 221 Burwood Highway, Burwood 3125, Australia. ${ }^{2}$ Medical and Clinical Affairs, Satellite Healthcare, San Jose, CA, USA. ${ }^{3}$ School of Nursing and Midwifery, Deakin University, Burwood, VIC, Australia. ${ }^{4}$ Department of Renal Medicine, Eastern Health Clinical School, Melbourne, VIC, Australia.}

Received: 4 April 2017 Accepted: 4 September 2017

Published online: 11 September 2017

\section{References}

1. National Kidney Foundation. K/DOQI clinical practice guidelines for chronic kidney disease: evaluation, classification, and stratification. New York: National Kidney Foundation; 2002.

2. Global Facts. About Kidney Disease [https://www.kidney.org/kidneydisease/ global-facts-about-kidney-disease].

3. USRDS 2013 Annual data report. atlas of end-stage renal disease in the United States [https://pharm.ucsf.edu/kidney/need/statistics].

4. Kidney Health Australia. Chronic kidney disease (CKD) management in general practice. Melbourne: Kidney Health Australia; 2012.

5. ANZDATA Registry. ANZDATA Registry 37th Annual Report. In. Edited by Registry AaNZDaT. Adelaide, Australia; 2014.

6. Johansen KL, Shubert T, Doyle J, Soher B, Sakkas GK, Kent-Braun JA. Muscle atrophy in patients receiving hemodialysis: effects on muscle strength, muscle quality, and physical function. Kidney Int. 2003;63(1):291-7.

7. Kim JK, Choi SR, Choi MJ, Kim SG, Lee YK, Noh JW, Kim HJ, Song YR. Prevalence of and factors associated with sarcopenia in elderly patients with end-stage renal disease. Clin Nutr. 2014:33(1):64-8.

8. Johansen KL. Exercise and dialysis. Hemodial Int. 2008;12(3):290-300
9. Johansen KL, Chertow GM, da Silva M, Carey S, Painter P. Determinants of physical performance in ambulatory patients on hemodialysis. Kidney Int. 2001;60(4):1586-91.

10. Tentori F, Elder SJ, Thumma J, Pisoni RL, Bommer J, Fissell RB, Fukuhara S, Jadoul M, Keen ML, Saran R, et al. Physical exercise among participants in the dialysis outcomes and practice patterns study (DOPPS): correlates and associated outcomes. Nephrol Dial Transplant. 2010;25(9):3050-62.

11. Hsieh RL, Lee WC, Chang CH. Maximal cardiovascular fitness and its correlates in ambulatory hemodialysis patients. Am J Kidney Dis. 2006;48(1):21-7.

12. Blake C, O'Meara YM. Subjective and objective physical limitations in high-functioning renal dialysis patients. Nephrol Dial Transplant. 2004;19(12):3124-9.

13. Sterky E, Stegmayr B. Elderly patients on haemodialysis have $50 \%$ less functional capacity than gender-and age-matched healthy subjects. Scand J Urol Nephrol. 2005;39(5):423-30.

14. Knight EL, Ofsthun N, Teng M, Lazarus JM, Curhan GC. The association between mental health, physical function, and hemodialysis mortality. Kidney Int. 2003;63(5):1843-51.

15. Heiwe S, Clyne N, Tollback A, Borg K. Effects of regular resistance training on muscle histopathology and morphometry in elderly patients with chronic kidney disease. Am J Phys Med Rehabil. 2005;84(11):865-74.

16. Heiwe S, Tollback A, Clyne N. Twelve weeks of exercise Traininglncreases muscle function and walking capacity in elderly Predialysis patients and healthy subjects. Nephron. 2001;88(1):48-56.

17. Greenwood SA, Lindup H, Taylor K, Koufaki P, Rush R, Macdougall IC, Mercer $\mathrm{TH}$. Evaluation of a pragmatic exercise rehabilitation programme in chronic kidney disease. Nephrol Dial Transplant. 2012;27((suppl 3)):iii126-34.

18. van den Ham EC, Kooman JP, Schols AM, Nieman FH, Does JD, Akkermans MA, Janssen PP, Gosker HR, Ward KA, MacDonald JH, et al. The functional, metabolic, and anabolic responses to exercise training in renal transplant and hemodialysis patients. Transplantation. 2007;83(8):1059-68.

19. Koufaki P, Greenwood SA, Macdougall IC, Mercer TH. Exercise therapy in individuals with chronic kidney disease: a systematic review and synthesis of the research evidence. Annu Rev Nurs Res. 2013;31(1):235-75.

20. Smart NA, Williams AD, Levinger I, Selig S, Howden E, Coombes JS, Fassett RG. Exercise \& Sports Science Australia (ESSA) position statement on exercise and chronic kidney disease. J Sci Med Sport. 2013;16(5):406-11.

21. Heiwe $\mathrm{S}$, Jacobson SH. Exercise training for adults with chronic kidney disease. Cochrane Database Syst Rev. 2011;10(10):CD003236.

22. Konstantinidou E, Koukouvou G, Kouidi E, Deligiannis A, Tourkantonis A Exercise training in patients with end-stage renal disease on hemodialysis: comparison of three rehabilitation programs. J Rehabil Med. 2002;34(1):40-5

23. Bennett PN, Daly RM, Fraser SF, Haines T, Barnard R, Ockerby C, Kent B. The impact of an exercise physiologist coordinated resistance exercise program on the physical function of people receiving hemodialysis: a stepped wedge randomised control study. BMC Nephrol. 2013;14(1):204.

24. Johansen KL. Exercise in the end-stage renal disease population. J Am Soc Nephrol. 2007:18(6):1845-54.

25. Abe T, Kearns CF, Sato Y. Muscle size and strength are increased following walk training with restricted venous blood flow from the leg muscle, Kaatsu-walk training. J Appl Physiol (1985). 2006;100(5):1460-6.

26. Loenneke JP, Wilson GJ, Wilson JM. A mechanistic approach to blood flow occlusion. Int J Sports Med. 2010;31(1):1-4.

27. Fahs CA, Loenneke JP, Thiebaud RS, Rossow LM, Kim D, Abe T, Beck TW, Feeback DL, Bemben DA, Bemben MG. Muscular adaptations to fatiguing exercise with and without blood flow restriction. Clin Physiol Funct Imaging. 2015;35(3):167-76.

28. Loenneke JP, Wilson JM, Marin PJ, Zourdos MC, Bemben MG. Low intensity blood flow restriction training: a meta-analysis. Eur J Appl Physiol. 2012; 112(5):1849-59.

29. Abe T, Fujita S, Nakajima T, Sakamaki M, Ozaki H, Ogasawara R, Sugaya M, Kudo M, Kurano M, Yasuda T, et al. Effects of low-intensity cycle training with restricted leg blood flow on thigh muscle volume and VO2MAX in young men. J Sports Sci Med. 2010;9(3):452-8.

30. Takarada Y, Takazawa H, Sato Y, Takebayashi S, Tanaka Y, Ishii N. Effects of resistance exercise combined with moderate vascular occlusion on muscular function in humans. J Appl Physiol (1985). 2000;88(6):2097-106.

31. Sumide T, Sakuraba K, Sawaki K, Ohmura H, Tamura Y. Effect of resistance exercise training combined with relatively low vascular occlusion. J Sci Med Sport. 2009;12(1):107-12 
32. Ozaki H, Sakamaki M, Yasuda T, Fujita S, Ogasawara R, Sugaya M, Nakajima T, Abe T. Increases in thigh muscle volume and strength by walk training with leg blood flow reduction in older participants. J Gerontol A Biol Sci Med Sci. 2011;66(3):257-63.

33. Abe T, Sakamaki M, Fujita S, Ozaki H, Sugaya M, Sato Y, Nakajima T. Effects of low-intensity walk training with restricted leg blood flow on muscle strength and aerobic capacity in older adults. J Geriatr Phys Ther. 2010;33(1):34-40.

34. Ozaki H, Miyachi M, Nakajima T, Abe T. Effects of 10 weeks walk training with leg blood flow reduction on carotid arterial compliance and muscle size in the elderly adults. Angiology. 2011;62(1):81-6.

35. Ozaki H, Loenneke JP, Thiebaud RS, Stager JM, Abe T. Possibility of leg muscle hypertrophy by ambulation in older adults: a brief review. Clin Interv Aging. 2013;8:369-75.

36. Gormley SE, Swain DP, High R, Spina RJ, Dowling EA, Kotipalli US, Gandrakota R. Effect of intensity of aerobic training on VO2max. Med Sci Sport Exer. 2008;40(7):1336

37. Park S, Kim JK, Choi HM, Kim HG, Beekley MD, Nho H. Increase in maximal oxygen uptake following 2-week walk training with blood flow occlusion in athletes. Eur J Appl Physiol. 2010;109(4):591-600.

38. Staunton CA, May AK, Brandner CR, Warmington SA. Haemodynamics of aerobic and resistance blood flow restriction exercise in young and older adults. Eur J Appl Physiol. 2015:1-10.

39. Nakajima T, Kurano M, lida H, Takano H, Oonuma H, Morita T, Meguro K Sato $Y$, Nagata T. Use and safety of KAATSU training: results of a national survey. Int J KAATSU Training Res. 2006;2(1):5-13.

40. McEwen JA, Kelly DL, Jardanowski T, Inkpen K. Tourniquet safety in lower leg applications. Orthop Nurs. 2002;21(5):61-2.

41. Koufaki $\mathrm{P}$, Kouidi E. Current best evidence recommendations on measurement and interpretation of physical function in patients with chronic kidney disease. Sports Med. 2010;40(12):1055-74.

42. Cheema B, Abas H, Smith B, O'Sullivan A, Chan M, Patwardhan A, Kelly J, Gillin A, Pang G, Lloyd B, et al. Progressive exercise for anabolism in kidney disease (PEAK): a randomized, controlled trial of resistance training during hemodialysis. J Am Soc Nephrol. 2007;18(5):1594-601.

43. DePaul V, Moreland J, Eager T, Clase CM. The effectiveness of aerobic and muscle strength training in patients receiving hemodialysis and EPO: a randomized controlled trial. Am J Kidney Dis. 2002;40(6):1219-29.

44. Garber CE, Blissmer B, Deschenes MR, Franklin BA, Lamonte MJ, Lee IM, Nieman DC, Swain DP. American College of Sports M: American College of Sports Medicine position stand. Quantity and quality of exercise for developing and maintaining cardiorespiratory, musculoskeletal, and neuromotor fitness in apparently healthy adults: guidance for prescribing exercise. Med Sci Sports Exerc. 2011;43(7):1334-59.

45. Antlanger M, Hecking M, Haidinger M, Werzowa J, Kovarik JJ, Paul G, Eigner M, Bonderman D, Horl WH, Saemann MD. Fluid overload in hemodialysis patients: a cross-sectional study to determine its association with cardiac biomarkers and nutritional status. BMC Nephrol. 2013;14:266.

46. Agarwal R, Peixoto AJ, Santos SF, Zoccali C. Pre- and postdialysis blood pressures are imprecise estimates of interdialytic ambulatory blood pressure. Clin J Am Soc Nephrol. 2006;1(3):389-98.

47. Headley S, Germain M, Wood R, Joubert J, Milch C, Evans E, Cornelius A, Brewer B, Taylor B, Pescatello LS. Blood pressure response to acute and chronic exercise in chronic kidney disease. Nephrology (Carlton). 2017;22(1):72-8.

48. Painter P, Moore G, Carlson L, Paul S, Myll J, Phillips W, Haskell W. Effects of exercise training plus normalization of hematocrit on exercise capacity and health-related quality of life. Am J Kidney Dis. 2002;39(2):257-65.

49. Mustata S, Groeneveld S, Davidson W, Ford G, Kiland K, Manns B. Effects of exercise training on physical impairment, arterial stiffness and health-related quality of life in patients with chronic kidney disease: a pilot study. Int Urol Nephrol. 2011;43(4):1133-41.

50. Toyama K, Sugiyama S, Oka H, Sumida H, Ogawa H. Exercise therapy correlates with improving renal function through modifying lipid metabolism in patients with cardiovascular disease and chronic kidney disease. J Cardiol. 2010;56(2):142-6.

51. Borg GA. Psychophysical bases of perceived exertion. Med Sci Sports Exerc. 1982;14(5):377-81.

52. Padilla J, Krasnoff J, Da Silva M, Hsu CY, Frassetto L, Johansen KL, Painter P. Physical functioning in patients with chronic kidney disease. J Nephrol. 2008;21(4):550-9.
53. Younger ASE, McEwen JA, Inkpen K. Wide contoured thigh cuffs and automated limb occlusion measurement allow lower tourniquet pressures. Clin Orthop Relat Res. 2004;428:286-93.

54. Brandner CR, Warmington SA. Delayed onset muscle soreness and perceived exertion following blood flow restriction exercise. J Strength Cond Res. 2017;

55. May AK, Brandner CR, Warmington SA. Hemodynamic responses are reduced with aerobic compared with resistance blood flow restriction exercise. Physiol Rep. 2017:5(3).

56. Kouidi E, Karagiannis V, Grekas D, lakovides A, Kaprinis G, Tourkantonis A Deligiannis A. Depression, heart rate variability, and exercise training in dialysis patients. Eur J Cardiovasc Prev Rehabil. 2010;17(2):160-7.

57. American College of Sports Medicine. ACSM's guidelines for exercise testing and prescription. Philadelphia, PA: Lippincott Williams \& Wilkins; 2013.

58. Wathen D. Load assignment. In: Essentials of strength training and conditioning; 1994. p. 435-46.

59. Jones CJ, Rikli RE, Beam WC. A 30-s chair-stand test as a measure of lower body strength in community-residing older adults. Res Q Exerc Sport. 1999;70(2):113-9.

60. Overend T, Anderson C, Sawant A, Perryman B, Locking-Cusolito H. Relative and absolute reliability of physical function measures in people with endstage renal disease. Physiother Can. 2010;62(2):122-8.

61. Shumway-Cook A, Brauer S, Woollacott M. Predicting the probability for falls in community-dwelling older adults using the timed up \& go test. Phys Ther. 2000;80(9):896-903.

62. Mesquita R, Janssen DJ, Wouters EF, Schols JM, Pitta F, Spruit MA. Withinday test-retest reliability of the timed up \& go test in patients with advanced chronic organ failure. Arch Phys Med Rehabil. 2013;94(11):2131-8.

63. Botolfsen P, Helbostad JL, Moe-Nilssen R, Wall JC. Reliability and concurrent validity of the expanded timed up-and-go test in older people with impaired mobility. Physiother Res Int. 2008;13(2):94-106.

64. Steffen TM, Hacker TA, Mollinger L. Age- and gender-related test performance in community-dwelling elderly people: six-minute walk test, berg balance scale, timed up \& go test, and gait speeds. Phys Ther. 2002;82(2):128-37.

65. Enright PL, McBurnie MA, Bittner $V$, Tracy RP, McNamara R, Arnold A, Newman AB, Cardiovascular Health S. The 6-min walk test: a quick measure of functional status in elderly adults. Chest. 2003;123(2):387-98.

66. Holub C, Lamont M, Lombardo A, Pence T, Schultz G, Tepper S. The reliability of the six-minute walk test in patients with end-stage renal disease. Acute Care Perspect. 2002;11(1):8-11.

67. Park SM, Ahn SJ, Lee JE, Kim YS, Lee SH, Moon HS, Chang YS, Bang BK. Validity and utility of 6-minute-walk test and WALK (walking-stair-climbing) test to assess the exercise capacity in hemodialysis patients. Korean J Nephrol. 2001;20(3):438-46.

68. Stewart AL, Mills KM, King AC, Haskell WL, Gillis D, Ritter PL. CHAMPS physical activity questionnaire for older adults: outcomes for interventions. Med Sci Sports Exerc. 2001:33(7):1126-41.

69. Collins ES, Witt J, Bausewein C, Daveson BA, Higginson IJ, Murtagh FE. A systematic review of the use of the palliative care outcome scale and the support team assessment schedule in palliative care. J Pain Symptom Manag. 2015;50(6):842-53. e819

70. Van Craenenbroeck AH, Van Craenenbroeck EM, Van Ackeren K, Hoymans VY, Verpooten GA, Vrints CJ, Couttenye MM. Impaired vascular function contributes to exercise intolerance in chronic kidney disease. Nephrol Dial Transplant. 2016;31(12):2064-72.

71. lida H, Nakajima T, Kurano M, Yasuda T, Sakamaki M, Sato Y, Yamasoba T, Abe T. Effects of walking with blood flow restriction on limb venous compliance in elderly subjects. Clin Physiol Funct Imaging. 2011;31(6):472-6. 